\title{
The Incremental Value of SPECT/CT in Characterizing Solitary Spine Lesions
}

\author{
Basit Iqbal ${ }^{1-3}$, Geoffrey M. Currie ${ }^{1,2,4}$, Janelle M. Wheat ${ }^{1,2,4}$, Hassan Raza ${ }^{3}$, and Hosen Kiat $^{1,2,4}$ \\ ${ }^{1}$ Faculty of Science, Charles Sturt University, Wagga Wagga, Australia; ${ }^{2}$ Centre for Research in Complex Systems, Charles Sturt \\ University, Wagga Wagga, Australia; ${ }^{3}$ Atomic Energy Medical Centre, Jinnah Postgraduate Medical Centre, Karachi, Pakistan; and \\ ${ }^{4}$ Australian School of Advanced Medicine, Macquarie University, Sydney, Australia
}

\begin{abstract}
The purpose of this study was to investigate the incremental value and diagnostic impact of SPECT/CT in patients who had a solitary spinal lesion on a bone scan. Methods: A prospective study was performed on 80 patients (50 with known cancer) who underwent $99 \mathrm{~m}$ Tc-methylene diphosphonate whole-body planar bone scintigraphy and had a solitary spinal lesion. These lesions were then further evaluated using SPECT/CT. Results: Lesions were localized to the vertebral body in 38 patients (47.5\%), pedicle in 15 (18.8\%), facet joint in 15 (18.8\%), transverse process in $2(2.5 \%)$, spinous process in $2(2.5 \%)$, lamina in $3(3.8 \%)$, and end plates in $5(6.2 \%)$. Although the specificity of planar bone scans was excellent $(100 \%)$, sensitivity was only $6.1 \%$ but increased to $78.8 \%$ after the addition of SPECT/CT. The results provide evidence of a substantial incremental increase in diagnostic accuracy using SPECT/CT over planar imaging alone for patients in whom a solitary spinal lesion is noted. Conclusion: The addition of SPECT/CT significantly reduced the number of false-negative results and increased the number of true-positive results. SPECT/CT also reduced the number of equivocal reports. A definitive diagnosis was given for most patients, indicating improved diagnostic confidence with the addition of SPECT/CT, compared with planar imaging alone, in patients with solitary spinal lesions.
\end{abstract}

Key Words: bone scan; SPECT; spine lesion; SPECT/CT; metastases

J Nucl Med Technol 2011; 39:201-207

DOI: 10.2967/jnmt.111.088351

B one scintigraphy has, for several decades, provided a highly sensitive, reliable, noninvasive method in the detection of focal bone disease (1). These attributes are complemented by its widespread availability, well-established safety record, moderate cost, extensive clinical experience, and well-documented efficacy in a broad range of diseases. Advances in instrumentation (resolution and sensitivity) and techniques (SPECT and

\footnotetext{
Received Jan. 21, 2011; revision accepted Apr. 15, 2011.

For correspondence or reprints contact: Geoffrey M. Currie, Faculty of Science, Locked Bag 588, Charles Sturt University, Wagga Wagga 2678, Australia.

E-mail: gcurrie@csu.edu.au

Published online Jul. 27, 2011.

COPYRIGHT @ 2011 by the Society of Nuclear Medicine, Inc.
}

more recently SPECT/CT), combined with the limitations of other imaging modalities (e.g., cost, availability, radiation dose, and lack of physiologic detail) have ensured that bone scintigraphy remains the preferred procedure in diagnostic algorithms for detection and characterization of bone lesions of various pathologic origins.

Bone metastases are by far the most common malignant bone tumors seen in adults (2). Bone metastases may occur with virtually all malignancies but are most common in carcinomas of the breast (47\%-85\%), lung (32\%), prostate $(54 \%-85 \%)$, kidney $(33 \%-40 \%)$, and thyroid $(28 \%-60 \%)$ (2). The spine is the most common site of skeletal metastases (39\%) because of the abundant vascularization and red bone marrow (2).

Most bone metastases result from hematogenous dissemination of cancer cells. Micrometastases are present in the bone marrow in $25 \%-75 \%$ of patients with common malignancies (3). The mechanism of development and growth of bone metastases is a multistep process, which requires complex interactions between the metastatic cells and the tissue. Metastases do not affect all the bones with the same pattern and frequency but generally prefer the spine and pelvis. Besides the molecular and biologic characteristics of both neoplastic and normal cells, other factors may play a role in the skeletal localization of malignant cells, such as the vascular methods of spreading, the speed, and the amount of blood flow toward specific areas (3). Bone constitutes a well-defined microcompartment supplied primarily by the bloodstream. In bone marrow, the particular capillary structures and the reduction of the speed of blood may favor the seeding of metastatic cells. Vascular sinusoids are lined by endothelial cells with 6-nm fenestrae and lack a basement membrane (3).

Because the spine represents the preferential site of metastatic skeletal spread, it is important to know how the blood supply is distributed to the vertebral bone through the vertebral arterial and venous plexuses. Batson (4) first described the evidence of a venous connection between the periprostatic and lumbar plexuses in 1940. The author evidenced the existence of a network of longitudinal, valueless vessels, running parallel to the vertebral column and forming countless anastomoses to the sinusoidal structure of the vertebral mar- 
row and epidural venous channels. The evidence suggested that, under increased abdominal pressure, metastatic prostate cells could reach the axial skeleton by retrograde hematogenous spreading and thus without passing the first anatomic filter (the lungs). More recently, Suzuki et al. (5) established the exact intraabdominal hemodynamic pressure necessary to make blood draining the prostate flow into the vertebral veins in animal models. Other studies have reported that this network of venous vessels may be wider and involve more blood areas. Indeed, it is possible to show the presence of links between Batson's plexus and the azygos-hemiazygos system and internal mammary system, by means of lateral thoracic and intercostal veins (3). These links could explain the possibility of metastasis to the spine even of tumor cells deriving from organs other than the prostate, such as breast and lung (3).

In the nuclear medicine department, bone scans for assessment of skeletal metastases typically use a whole-body sweep technique with $\gamma$-camera systems equipped with high- or ultra-high-resolution collimators, allowing excellent image quality. When a suggestive focal abnormality is detected, additional static projections or SPECT may be indicated to better delineate the lesion (6).

SPECT enables more accurate localization of tracer accumulation, especially in anatomic regions that are otherwise difficult to interpret because of complex skeletal architecture (such as the spine, pelvis, or skull) or under- or overlying structures. Moreover, the ability to view biodistribution in multiple sections (e.g., transverse, sagittal, and coronal) means that more precise definition of the location and extent of lesions can be determined, particularly in relation to traversing joints. Furthermore, tracer uptake on planar images may appear similar for different malignant and benign conditions, and thus, SPECT affords the potential to improve diagnostic specificity on the basis of more accurate differentiation of the cause of uptake (1). Nonetheless, in many instances (e.g., solitary lesions in the axial skeleton), correlation with high-quality anatomic images (e.g., CT) may be required for diagnosis. Visualization of the exact anatomic localization and the morphology of underlying bone disease may improve the specificity of bone scans considerably.

Extensive work has been performed on image fusion software during the past $20 \mathrm{y}$ (7). Accurate image fusion, although relatively easy in the brain and extremities because of the rigid structures, is more challenging in the thorax and abdomen (7). Patient motion, organ motion, and, particularly in the context of bone imaging, differences in the curvature and shape of imaging tables may lead to differences in positioning of the spine and other structures. Several initial reports on fusion imaging addressed the benefit of the fusion of SPECT data with CT performed on a separate camera at a different time. In theory, the concept of software fusion is fascinating, but in practice, image fusion may be difficult to achieve. In some situations, particularly if patients present with coexisting disease (e.g., degenerative bone disease, in-
TABLE 1

Criteria for Lesion Characterization

\begin{tabular}{cc}
\hline \multicolumn{1}{c}{ Benign } & Malignant \\
\hline Facet joint & Body and pedicle \\
Costovertebral joint & $\begin{array}{c}\text { Photon-deficient } \\
\text { area (lytic area) }\end{array}$ \\
$\begin{array}{l}\text { Involvement of } 2 \text { adjacent } \\
\text { vertebrae or endplates } \\
\text { of vertebrae }\end{array}$ & \\
\hline
\end{tabular}

flammation, or metabolic disease), the results may be unsatisfactory. SPECT/CT on a hybrid system, even with low-dose CT, overcomes many of these limitations by scanning the patient sequentially on the same imaging table to improve diagnostic and localization accuracy.

Detection of skeletal metastases is clinically important because of associated symptoms, complications (e.g., pathologic fractures), and significance for staging, treatment, and prognosis. Differentiating between benign and metastatic bone lesions, however, can be difficult with planar scintigraphy alone (8). Compared with planar scintigraphy, SPECT increases image contrast and improves lesion detection and localization (9).

\section{MATERIALS AND METHODS}

The aim of this investigation was to assess the benefit of combined SPECT/CT for classification of solitary lesions in the vertebrae. The investigation was approved by an institutional ethics committee.

The prospective study included patients who were referred for a bone scan (deletion) during 2008 and who were diagnosed with a solitary lesion in the vertebrae on the planar images. All patients also underwent SPECT/CT on the same day as the planar study. A follow-up planar and SPECT/CT bone scan was performed 3 mo later, and the results were used as the reference standard.

Lesions were classified as being consistent with the appearance of either benign or malignant disease on the basis of the criteria described in Table 1. Lesions that could not be categorized as either benign or malignant were classified as indeterminate. Each study was independently interpreted by 2 nuclear medicine physicians who were unaware of the patients' clinical history and test results. The interpreting physicians were also unaware of each other's interpretation, and discordant classification was determined to be indeterminate. Interpretation was undertaken for planar and SPECT/CT images separately and sequentially.

TABLE 2

Comparative Results for Both Study Populations at Initial Imaging Session

\begin{tabular}{lcccc}
\hline Imaging type & Indeterminate & Benign & Malignant & Total \\
\hline Planar & $51(63.8 \%)$ & $27(33.8 \%)$ & $2(2.5 \%)$ & 80 \\
SPECT/CT & $11(13.8 \%)$ & $39(48.8 \%)$ & $30(37.5 \%)$ & 80 \\
\hline
\end{tabular}


TABLE 3

Outcomes for Baseline and Follow-up Planar Imaging and SPECT/CT in Oncology Cohort

\begin{tabular}{lcccc}
\hline Imaging type & Indeterminate & Benign & Malignant & Total \\
\hline Baseline planar & $26(52 \%)$ & $22(44 \%)$ & $2(4 \%)$ & 50 \\
Follow-up planar & $7(14 \%)$ & $20(40 \%)$ & $23(46 \%)$ & 50 \\
Baseline & $5(10 \%)$ & $17(34 \%)$ & $28(56 \%)$ & 50 \\
SPECT/CT & $5(10 \%)$ & $17(34 \%)$ & $28(56 \%)$ & 50 \\
Follow-up & $0(0 \%)$ & $17(34 \%)$ & $33(66 \%)$ & 50 \\
SPECT/CT & & & & \\
\hline
\end{tabular}

All patients were scanned using an Infinia Hawkeye lowdose (2.5 kVp) SPECT/CT system (GE Healthcare). After whole-body sweep acquisitions, planar imaging of the spine was performed using a $256 \times 256$ matrix for 1,000 kilocounts. SPECT/CT was performed through $360^{\circ}$ using $3^{\circ}$ angular sampling, a $128 \times 128$ matrix, and 15 s per step, with the patient supine. Data were processed using iterative reconstruction with $\mathrm{CT}$-based attenuation correction.

\section{RESULTS}

A total of 80 consecutive patients fulfilled the entry criteria. Fifty had known cancer (oncology cohort). Of those, 26 $(52 \%)$ had breast cancer, $12(24 \%)$ had prostate cancer, 6 (12\%) had lung cancer, $2(4 \%)$ had uterine cancer, $1(2 \%)$ had bladder cancer, 1 (2\%) had facial cancer, and $2(4 \%)$ had an unknown primary. The other 30 patients had no prior history of cancer (nononcology cohort). Of those, 15 (50\%) presented with backache, $7(23.3 \%)$ with trauma, and 5 (16.7\%) with infection, and $3(10 \%)$ were classified as miscellaneous. Lesions were localized to the vertebral body in 38 patients $(47.5 \%)$, pedicle in $15(18.8 \%)$, facet joint in $15(18.8 \%)$, transverse process in $2(2.5 \%)$, spinous process in $2(2.5 \%)$, lamina in $3(3.8 \%)$, and end plates in $5(6.2 \%)$. The results of image interpretation of the initial planar and SPECT images are summarized in Table 2.

In the oncology cohort $(n=50)$, the 3-mo follow-up provided improved demarcation of disease. Progression of disease was noted in 23 (46\%) of the patients, no change or the same disease pattern was seen in 17 (34\%) of the patients, and a change in intensity of the lesion was noted in
10 patients $(20 \%)$. The relative improvements in both planar imaging and SPECT can be noted in Table 3, and analysis of performance is summarized in Table 4.

In the 30 nononcology patients, SPECT/CT provided a diagnosis in $24(80 \%)$ patients, including 8 cases of osteomyelitis, 5 of posttraumatic injury, 4 of spondylosis, 3 of spondylolisthesis, and 2 each of facet joint osteoarthritis and metastasis. Planar imaging did not perform nearly as well, with just 5 diagnoses (17\%) (Table 5). If we assume, for the purposes of comparison, that SPECT/CT had no false-positive or false-negative results (unlikely to be true), then we can determine that, relative to $100 \%$ sensitivity and specificity for SPECT/CT, planar imaging would have a $20.8 \%$ sensitivity and a $100 \%$ specificity.

The following cases are representative of the entire sample and provide insight into the important role of SPECT/CT of the spine. More specifically, these cases highlight the advantage of SPECT/CT in more definitively identifying the disease and in differentiating between various diseases. Importantly, the cases provide examples in which the suspected disease was both upgraded to and downgraded from metastatic disease.

Case 1 was a $45-y$-old woman with a history of breast cancer, 1 mo of back pain, no history of trauma, and normal findings on radiography. Planar bone scanning demonstrated a subtle area of tracer accumulation, possibly indicating degeneration, in the region of the fourth lumbar vertebra (Fig. 1). SPECT/CT indicated a focal accumulation in the body of the vertebra consistent with early metastatic disease (Fig. 2). Three-month follow-up imaging demonstrated advancement of disease, including multiple spine, skull, and pelvic lesions, confirming the original SPECT/CT findings (deletion).

Case 2 was an 80-y-old man with a history of prostate cancer and lower backache for $15 \mathrm{~d}$. The patient had a prostate-specific antigen level of $727 \mathrm{ng} / \mathrm{dL}$. The planar bone scan demonstrated some mild changes suggestive of degenerative changes (Fig. 3); however, SPECT/CT revealed early metastases (Fig. 4).

In both the first and the second cases, on the basis of the planar scans the lesions could readily have been dismissed as degenerative because of their sites and location, but

TABLE 4

Performance Analysis for Planar Imaging and SPECT/CT in Oncology Cohort

\begin{tabular}{lccc}
\hline Index & Baseline planar & Baseline SPECT/CT & Follow-up planar \\
\hline True-negative & 17 & 15 & 17 \\
False-negative & 31 & 7 & 10 \\
True-positive & 2 & 26 & 23 \\
False-positive & 0 & 2 & 0 \\
Sensitivity (true-positive rate) & $6.1 \%$ & $78.8 \%$ & $69.7 \%$ \\
Specificity (true-negative rate) & $100 \%$ & $88.2 \%$ & $100 \%$ \\
Prevalence & $4 \%$ & $66 \%$ & $46 \%$ \\
Positive value of positive result & $100 \%$ & $92.9 \%$ & $100 \%$ \\
Positive value of negative result & $35.4 \%$ & $68.2 \%$ & $63.0 \%$ \\
\hline
\end{tabular}


TABLE 5

Comparison of Planar Imaging and SPECT/CT Outcomes in Nononcology Cohort

\begin{tabular}{lrr}
\hline Imaging type & Diagnosed & Undiagnosed \\
\hline Planar & $5(17 \%)$ & $25(83 \%)$ \\
SPECT/CT & $24(80 \%)$ & $6(20 \%)$ \\
\hline
\end{tabular}

SPECT/CT resulted in a change in diagnosis that affected patient management.

Case 3 was a 45-y-old man who presented with a suspected posttraumatic or degenerative change in the spine on planar images (Fig. 5). This finding was shown on CT to be multiple lytic lesions in multiple vertebrae (Fig. 6), and correlation with fusion (Fig. 7) revealed metastatic collapse of vertebrae in multiple myeloma. The skull radiograph showed a typical pepper-pot appearance. In this case, the patient presented with long-standing backache, and the planar bone scan was relatively unremarkable, with findings consistent with early degenerative changes. SPECT/CT, however, revealed a more sinister cause for the backache.

Case 4 was a 70-y-old man with a history of prostate cancer and trauma who presented with back pain. L2 radiopharmaceutical uptake on the planar bone scan (Fig. 8) was shown to be a posttraumatic vertebral body fracture on SPECT/CT (Fig. 9). In this case, given the patient's age and history and the pattern of the disease on the bone scan, metastasis was a consideration on the planar study. SPECT/ $\mathrm{CT}$ allowed delineation of a less sinister cause for the pain: posttraumatic changes.

The final case was a 52-y-old woman with lower back pain and no history of trauma. The planar bone scan showed diffuse L2 and L3 radiopharmaceutical uptake (Fig. 10) that was suggestive of disease secondary to osteoporosis. On

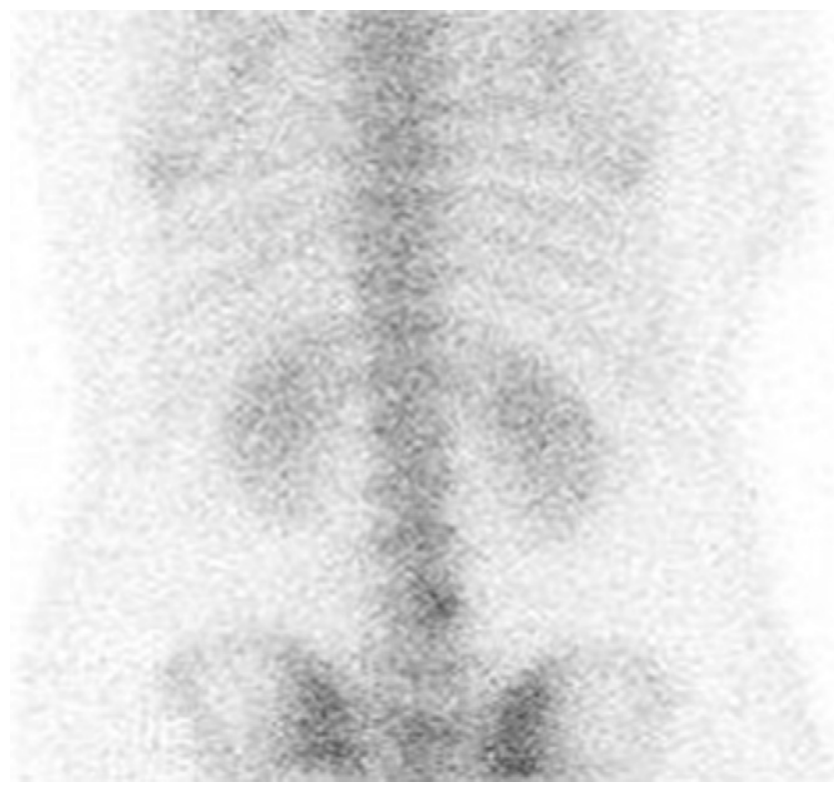

FIGURE 1. Baseline planar scan of spine.

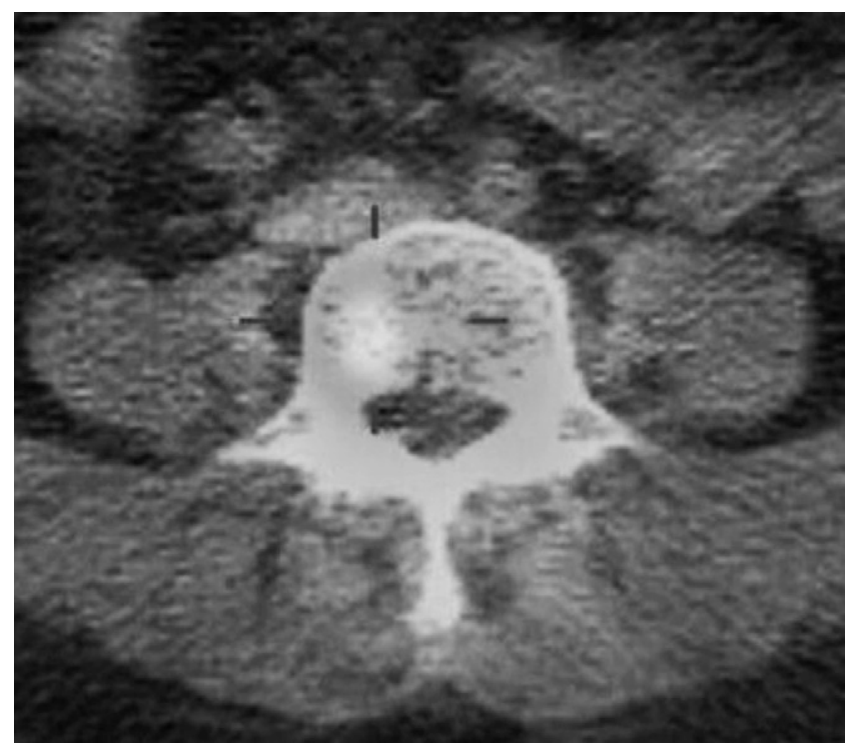

FIGURE 2. Baseline SPECT/CT scan. A color version of this figure is available as a supplemental file at http://tech.snmjournals.org.

SPECT/CT, this uptake was revealed to indicate intravertebral disk infection (Fig. 11). Subsequent work-up revealed it to be tuberculosis.

\section{DISCUSSION}

Our results demonstrated an improvement in the diagnostic efficacy of bone scanning with the addition of SPECT/CT,

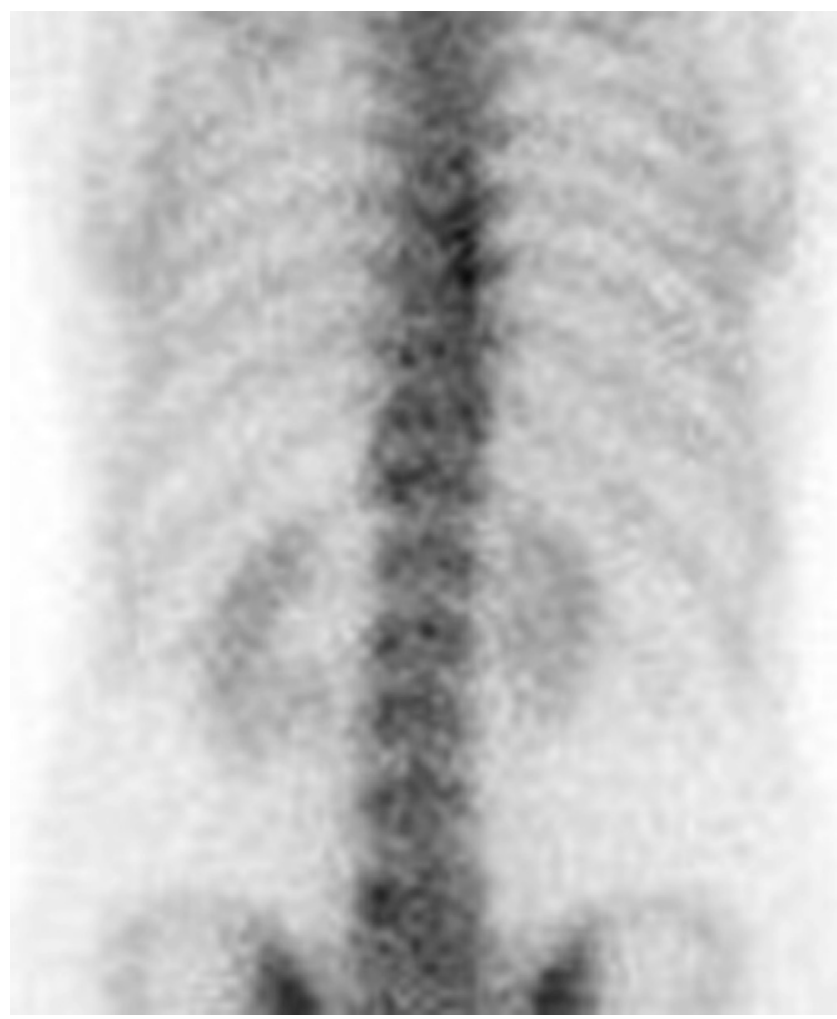

FIGURE 3. Bone scan showing L5 degenerative changes. 


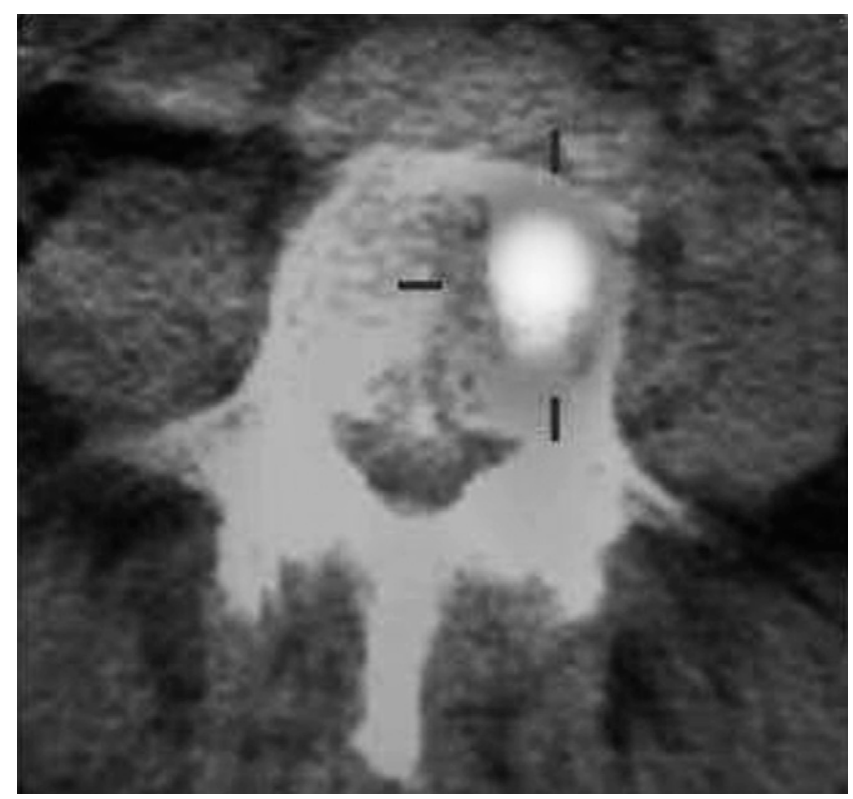

FIGURE 4. SPECT/CT scan showing early metastasis. A color version of this figure is available as a supplemental file at http:// tech.snmjournals.org.

for the investigation of solitary vertebral lesions. Specifically, the indeterminate outcome was reduced from $63.8 \%$ for planar imaging to $13.8 \%$ for SPECT/CT. The most important outcome was the improved characterization of

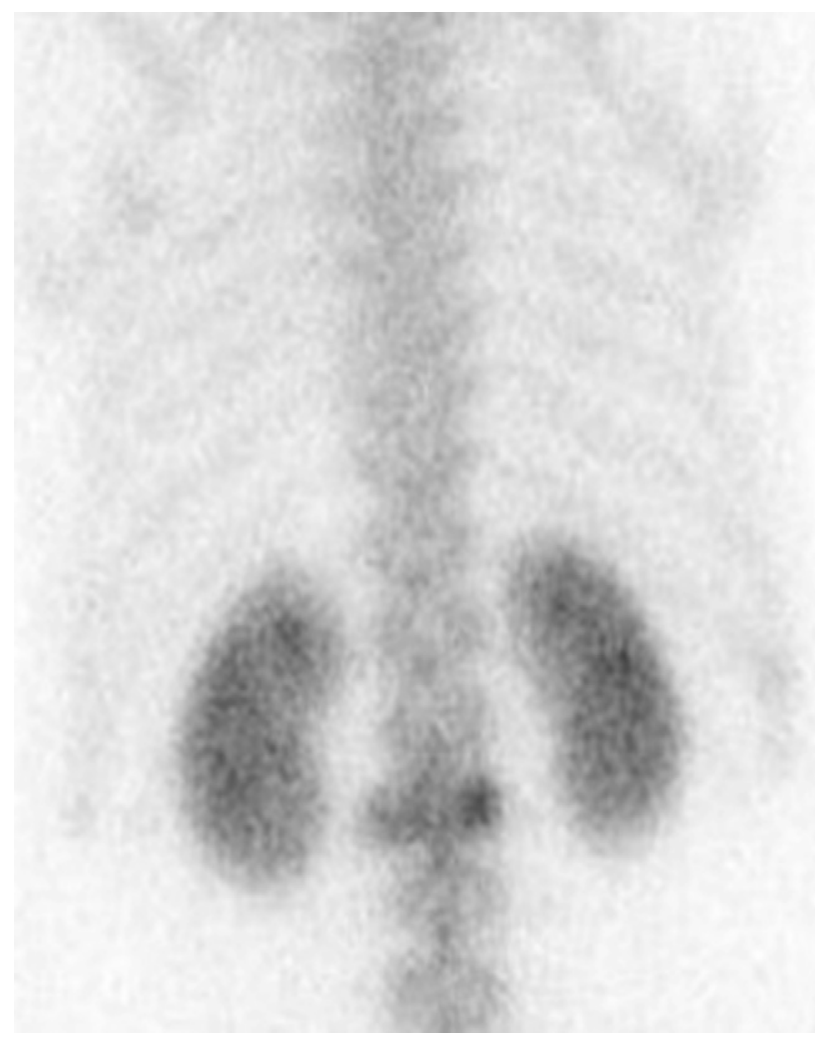

FIGURE 5. Bone scan suggesting posttraumatic or degenerative change.

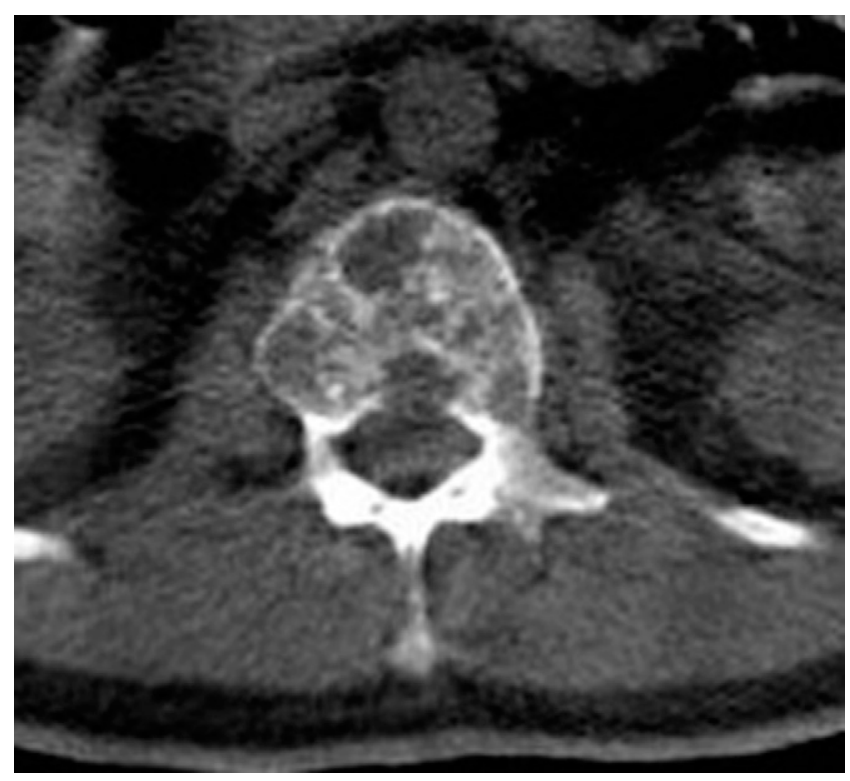

FIGURE 6. CT scan showing multiple lytic lesions.

spine lesions, with an increase in sensitivity from $6.1 \%$ to $78.8 \%$, despite a decrease in specificity from $10 \%$ to $88.2 \%$ for planar imaging and SPECT/CT, respectively. One key observation was the $46 \%$ of oncology patients in whom disease progression was noted during the 3-mo follow-up. Although this finding advocates the role of bone scanning in monitoring disease progression, it also provides a clear indication of the need for accurate and early diagnosis on the initial scan, since disease progression and prognosis may change substantially in a short time.

Sedonja et al. (10) performed planar scintigraphy and SPECT on 37 patients with lower back pain without known malignant lesions and on 38 patients with confirmed malignant disease. Overall, significantly more metastatic lesions

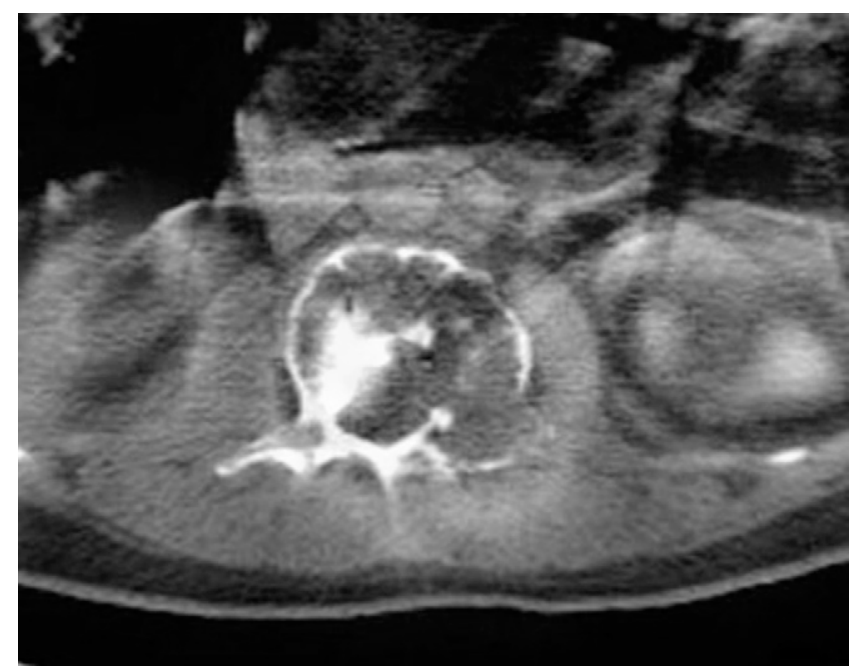

FIGURE 7. SPECT/CT scan highlighting metastatic disease. A color version of this figure is available as a supplemental file at http://tech.snmjournals.org. 


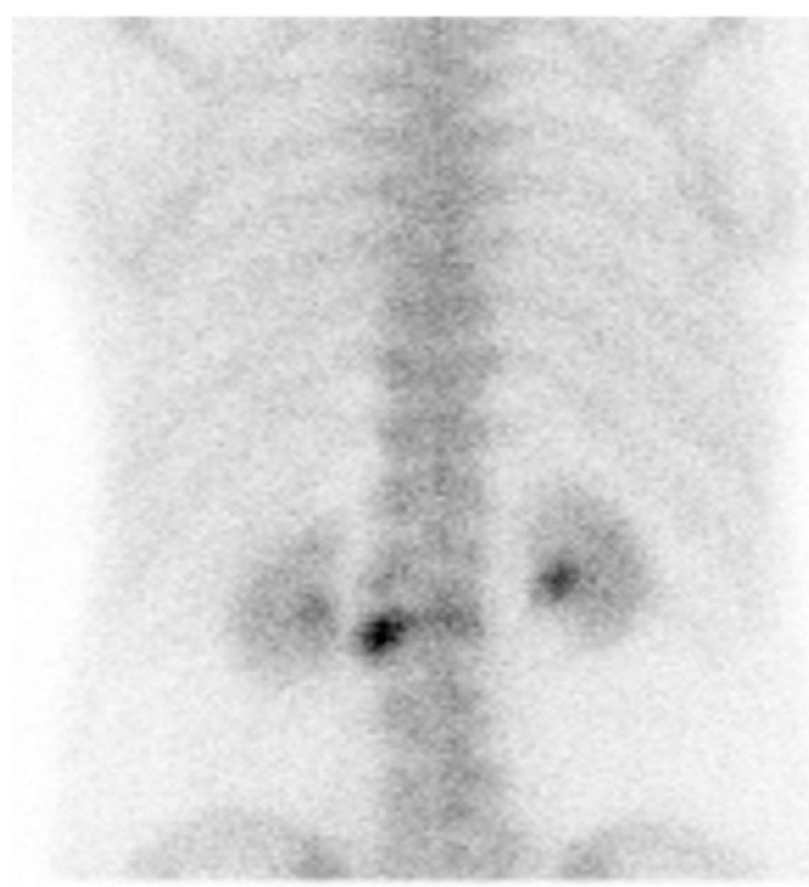

FIGURE 8. Bone scan showing L2 lesion.

were detected with SPECT (SPECT, 58/64 lesions; planar scintigraphy, 42/64 lesions; $P<0.01$ ).

Incidental focal tracer accumulation in patients with known malignancy can pose a clinically significant diagnostic dilemma, especially when there is only a solitary lesion (1). In a study at the Department of Nuclear Medicine at University Hospital Zurich, of the 42 lesions that were studied (in 37 patients), a specific diagnosis was made with planar scintigraphy in $64 \%$ of cases, with SPECT in $86 \%$, and with SPECT/CT in all cases (8). This observation is likely to ac-

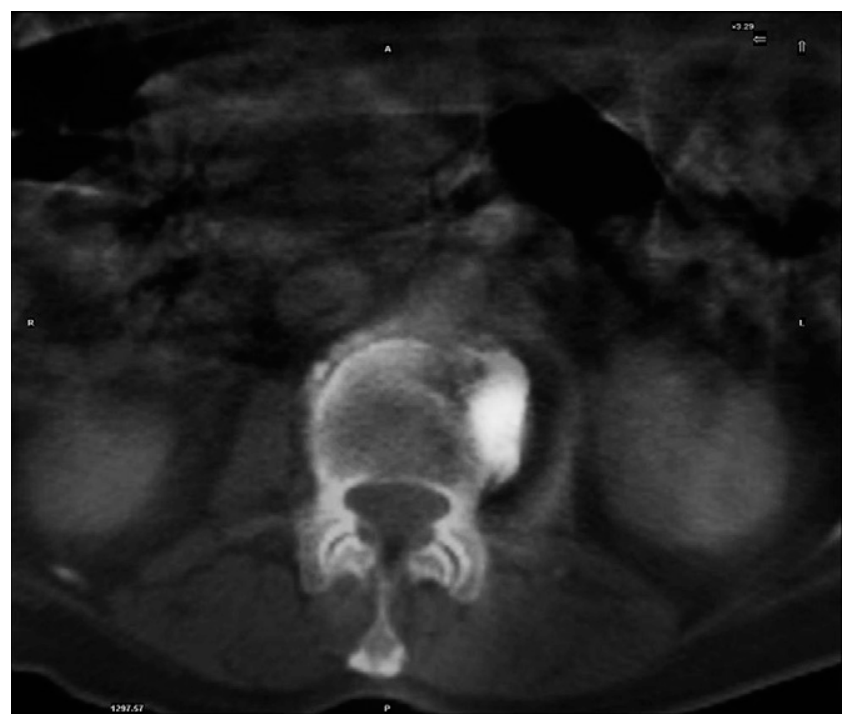

FIGURE 9. SPECT/CT scan showing vertebral body fracture. A color version of this figure is available as a supplemental file at http://tech.snmjournals.org.

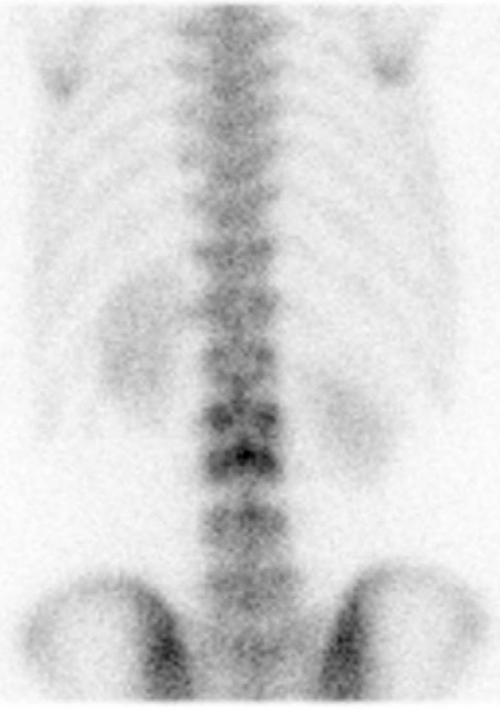

FIGURE 10. Planar bone scan.

count for the greater improvement reported in our investigation than reported by Sedonja et al. (10), highlighting the incremental benefit of SPECT/CT over SPECT alone, in comparison with planar bone scanning.

The results summarized in Table 4 provide evidence of a substantial incremental increase in diagnostic accuracy using SPECT/CT over planar imaging for patients in whom a solitary spinal lesion is noted. The use of SPECT/CT when there is some diagnostic ambiguity (especially when the spine is involved) can prevent misdiagnosis and, there-

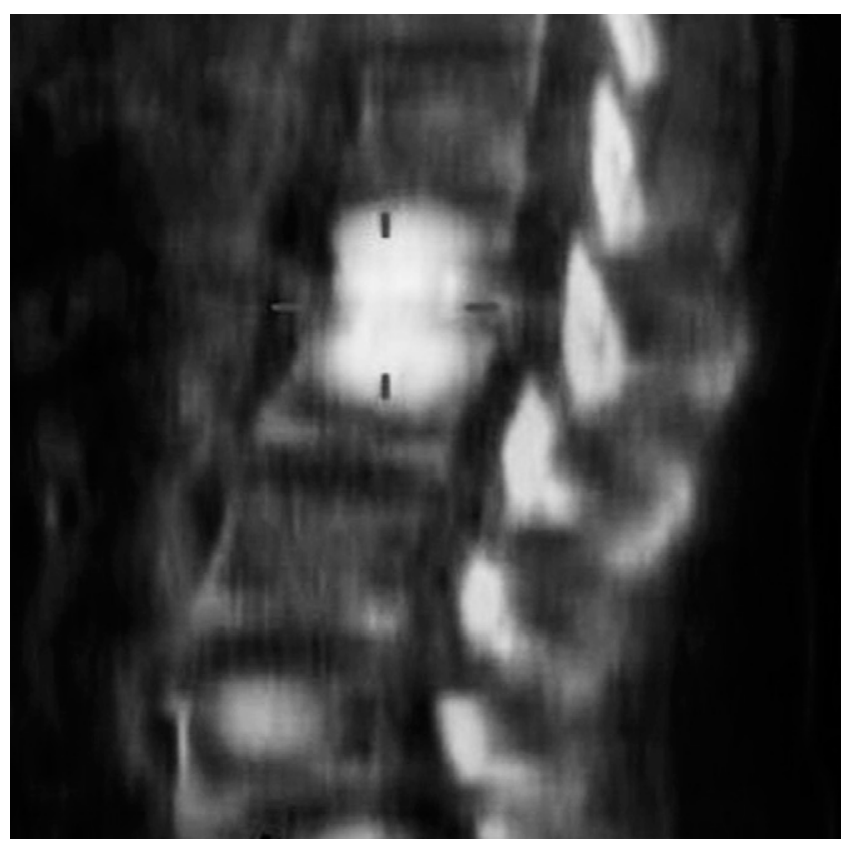

FIGURE 11. SPECT/CT scan showing intravertebral disk infection. A color version of this figure is available as a supplemental file at http://tech.snmjournals.org. 
fore, improve patient management. The regular use of SPECT/CT in such cases is further supported by the case examples provided. Nonetheless, the study and results are limited by the relatively small population and the lack of a histologic gold standard. These limitations should be addressed in future research, including more rigorous costeffectiveness analysis.

\section{CONCLUSION}

Our results indicate that the addition of localized SPECT/ CT for the assessment of indeterminate foci both enables a definitive diagnosis in most cases and improves diagnostic confidence. These results should encourage future research to assess the relative cost-effectiveness of SPECT/CT over planar or SPECT bone scintigraphy in the evaluation of solitary vertebral lesions in oncologic patients.

\section{ACKNOWLEDGMENT}

We thank Bashir Ahmed, formerly of the Atomic Energy Medical Centre, JPMC, Karachi, Pakistan, for contributing to data collection and reporting. No potential conflict of interest relevant to this article was reported.

\section{REFERENCES}

1. Horger M, Bares R. The role of single-photon emission computed tomography/ computed tomography in benign and malignant bone disease. Semin Nucl Med. 2006;36:286-294.

2. Taoka T, Mayr NA, Lee HJ, et al. Factors influencing visualization of vertebral metastases on MR imaging versus bone scintigraphy. AJR. 2001;176:1525-1530.

3. Savelli G, Maccauro M, De Deckere E, Bombardieri E. Bone scintigraphy and the added value of SPECT (single photon emission tomography) in detecting skeletal lesions. Q J Nucl Med. 2001;45:27-37.

4. Batson OV. Function of vertebral veins and their role in spread and metastases. Ann Surg. 1940;112:138-149.

5. Suzuki T, Kurokawa K, Okabe K, Ito K, Yamanaka H. Correlation between the prostatic vein and vertebral venous system under various conditions. Prostate. 1992;21:153-165.

6. Society of Nuclear Medicine Procedure Guideline for Bone Scintigraphy. SNM Web site. Available at: http://interactive.snm.org/docs/pg_ch34_0403.pdf. Accessed May 16, 2011.

7. Gnanasegaran G, Barwick T, Adamson K, Mohan H, Sharp D, Fogelman I. Multislice SPECT/CT in benign and malignant bone disease: when the ordinary turns into the extraordinary. Semin Nucl Med. 2009;39:431-442.

8. Strobel K, Burger C, Seifert B, Husarik DB, Soyka JD, Hany TF. Characterization of focal bone lesions in the axial skeleton: performance of planar bone scintigraphy compared with SPECT and SPECT fused with CT. AJR. 2007;188: W467-W474.

9. Kobayashi K, Kusota T, Nakai T, Ushijima Y, Nishimura T. Do short-time SPECT images of bone scintigraphy improve the diagnostic value in the evaluation of solitary lesions in the thoracic spine in patients with extraskeletal malignancies? Ann Nucl Med. 2005;19:557-566.

10. Sedonja I. The benefit of SPECT when added to planar scintigraphy in patients with bone metastases in the spine. Clin Nucl Med. 1999;24:407-413. 